\title{
The Influence of a Laboratory Testing Method on the Index of Density of Sand
}

\author{
Waldemar St. Szajna, Paulina Lechocka \\ Institute of Building Engineering University of Zielona Gora \\ Szafrana 1 Str., Zielona Gora, Poland \\ W.Szajna@ib.uz.zgora.pl; Paulina.Lechocka@gmail.com
}

\begin{abstract}
The paper presents the problem of evaluation of the index of density for sand by various laboratory methods. The parameter is well-suited for describing the state of coarse-grained soils (sand and gravel) because it is dimensionless and normalized. It takes values between $0 \div 1$. The index of density may be defined by maximum, minimum and apparent dry density, values of which should be evaluated in laboratory tests. Because of its definition, the parameter is very sensitive to measurement errors of dry density values. Even inconsiderable values of errors affect significantly the index of density. To estimate this sensitivity, a series of tests was performed. The tested material was classified as sub-angular, even-grade, medium quartz sand. Its maximum dry density was determined with the use of: Tapping Fork, Vibrating Table, Vibrating Hammer, Standard Proctor and Modified Proctor methods. The minimum values of dry density were determined with the use of Funnel method (spout diameter $10 \mathrm{~mm}$ and $12.5 \mathrm{~mm}$ ) and Inverting Cylinder - Kolbuszewski method. The total numbers of 35 maximum and 26 minimum dry density tests were performed. The tests results revealed significant differences in the values of the index of density evaluated by various standard methods. It may result in misunderstandings. Despite the same definition, values received by various national standard procedures differ so much that it is difficult to regard it as one parameter. The fact that engineers refer to this parameter with the same name, may lead to even greater confusion. The authors suggest that additional notation should be introduced in the symbol to clarify the methodology used.
\end{abstract}

Keywords: relative density, dry density evaluation methods, laboratory classification tests, vibratory compacting methods, granular soil

\section{Introduction}

The advancing international cooperation in technology means that national design standards and standards for material testing are adjusted to or even replaced with international standards. In Europe, it was the introduction of Eurocode 7 and related standards which significantly influenced the abovementioned process of modifying national standards in the field of geotechnics. As a result of these changes, engineers in various countries need to supplement their knowledge, but at the same time they may confront the existing soil testing procedures with the new methods.

In geotechnical design, soil strength and stiffness are the key parameters. Not only do most of these parameters depend on the type (nature) of the material (i.e. mineralogical composition, shape of grains, particle size distribution, etc.), but also on its state and structure (fabric and bonding). The state of soil, understood as a granular porous medium, may be described by a number of various parameters, of which the most commonly used are: porosity $(n)$, void ratio $(e)$, bulk density $(\rho)$, or dry density $\left(\rho_{d}\right)$. However, the above state parameters are not always convenient in use. For example, if the void ratio of sand is 0.6 , it is impossible to conclude whether it is in a loose or dense state. Moreover, for angular or poorly graded sand, this value will indicate its considerable density, whereas for well-rounded or well-graded sand it will suggests its loose state. The evaluation of the state of soil medium with the use of the above parameters $\left(n, e, \rho\right.$, or $\left.\rho_{d}\right)$ is possible if their extreme (minimum and maximum) values are known.

A parameter which is well-suited for the determination of the state of coarse-grained soils (sand and gravel) is the index of density $I_{D}=\left(e_{\max }-e\right) /\left(e_{\max }-e_{\min }\right)$. This is a dimensionless and normalized parameter, and it takes values between $0 \div 1$. As seen in the above formula, the definition of this index includes the minimum, maximum and the current values of void ratio. The significance of this parameter in geotechnical engineering results from the fact that the effective angle of internal friction $\phi^{\prime}$ correlates well with its value. 
Due to the difficulty in the determination of the void ratio in laboratory tests, it is convenient to write the definition of the index of density in the following form:

$$
I_{D}=\frac{\rho_{d \max }\left(\rho_{d}-\rho_{d \text { min }}\right)}{\rho_{d}\left(\rho_{d \text { max }}-\rho_{d \text { min }}\right)}
$$

where: $\rho_{d \min }, \rho_{d \max }$ and $\rho_{d}$ denotes minimum, maximum and apparent dry density respectively.

There are two approaches in the determination of the index of density in laboratory tests:

1. The first approach involves finding the minimum and the maximum dry density values. Both values are searched with the use of any arbitrary method returning best, i.e. the smallest and the largest values, respectively.

2. The second approach consists in applying methods which provide high reproducibility of results, even if they are not most effective. One method is used to find the minimum value of dry density, and another to find its maximum value. The obtained values are contractual since they depend on the method. Next, the apparent dry density value is determined for the examined soil.

The latter approach is applied in the standards of laboratory soil tests in individual countries.

American standard [1] specifies vibrating table method for $\rho_{d \max }$ determination and standard [2] defines funnel pouring, filled tube and inverting cylinder methods for $\rho_{d \min }$ evaluation. British standard [3], which Eurocode 7 [4] refers to, specifies vibrating hammer method for maximum dry density and inverting cylinder (Kolbuszewski) method for minimum dry density determination. German standard [5] defines tapping fork method of saturated soil sample for maximum dry density and funnel or spoon pouring methods for minimum dry density evaluation. Polish standard [6] specifies tapping fork method of oven-dried soil for maximum dry density and funnel pouring method for minimum dry density determination.

In the 1970s, a number of articles were devoted to laboratory methods for the determination of the index of density as well as the factors influencing this parameter. Youd [7] analysed the influence of particle shape and particle size distribution on sand density. A particularly considerable number of articles referred to soil compaction on the vibrating table. Brand [8] and Dobry and Whitman [9] analysed factors influencing the efficiency of soil compaction on the vibrating table. Tavens and La Rochelle [10] investigated errors affecting relative density measurements. A number of various methods for the determination of the minimum soil density were analysed, and the vibrating table method for the determination of the maximum soil density was studied. Prochaska and Drnevich [11] searched for an efficient method of the determination of the maximum dry density, and examined the following testing methods: Standard Proctor, Modified Proctor and Vibrating Hammer method. The Vibrating Hammer method proved to be fast and efficient. Yoshimi and Tohno [12] analysed factors affecting the reliability of relative density from the statistical point of view. The authors conclude that due to the definition (1) of the index of density, inconsiderable values of measurement errors affect considerably the error of the index density.

Considering the conclusion resulting from work [12] and the fact that in standard methods, the maximum and minimum values of soil density are contractual magnitudes (their values obtained on the basis of various standard procedures may differ), attention should be drawn to the fact that the values of the index of density for the same soil determined according to various national standard procedures may differ significantly one from another. For large differences, it would mean that, despite the same definition, in various countries, scientists and researchers use de facto different parameters, values of which do not fit to each other, and the fact that they are referred to with the same name may lead to even greater confusion. In the era of globalization and international exchange of services, the consequences of this could be serious. In order to evaluate the value of these differences, the authors tested the index of density of sand, applying a number of various standard procedures.

\section{Characteristics of the Analysed Sand and Description of Dry Density Testing Methods}

The tested material consisted of quartz sand derived in Droszkow, near Zielona Gora, western Poland. According to ISO [13] classification, it is medium sand $\left(d_{50}=0.36 \mathrm{~mm}\right)$ with sub-angular grains. The sand is even-grade, coefficient of uniformity $C_{U}=2.58$, coefficient of curvature $C_{C}=1.08$. It is a typical outwash sediment, characteristic for sandr areas occurring in western Poland. The particle size distribution curve of the sand is presented in Figure 1. 


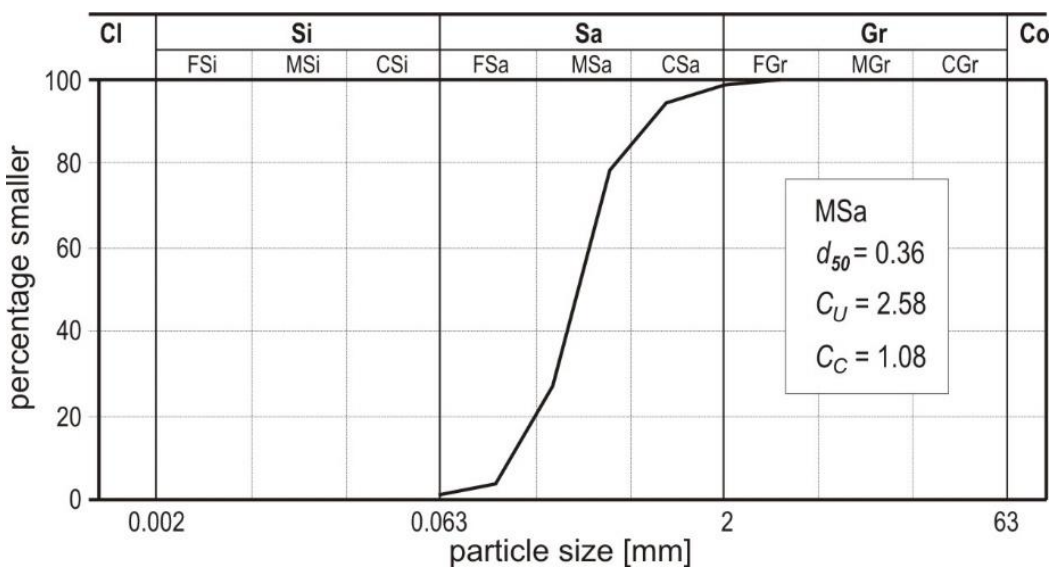

Fig. 1: Particle size distribution curve of Droszkow quartz outwash sand.

According to equation (1), the main task was to determine dry densities $\rho_{d \max }$ and $\rho_{d \min }$. The maximum dry density of sand was determined with the use of 5 methods. Three of them are typical methods used for the determination of the index of density: Tapping Fork [6], Vibrating Table [1] and Vibrating Hammer [3]. The other two, used for the determination of relative compaction, include: Standard Proctor and Modified Proctor methods. Proctor's tests were carried out because of their widespread usage for embankment compaction monitoring. The total number of 35 tests of $\rho_{d \max }$ was performed. Tab. 1 (points $1 \div 5$ ) presents a list of methods, including the used abbreviations, the number of tests performed, test conditions as well as references to respective standards. However, Tapping Fork and Vibration Table methods require additional comments. The TF test, where human factor influences considerably the obtained results, was performed by two laboratory workers. Each of them performed 5 tests. The VT test was performed not fully according to ASTM standard, which was marked with ' $\sim$ ' in the table. The essential differences were as follows: the measuring cylinders used in the test were of different dimensions than specified in ASTM standard, and the eccentric vibrating table was adopted from the process of concrete consistency control. The details of sand testing procedures with the TF and VT methods are presented in Szajna and Lechocka [14].

Table 1: Description of dry density tests.

\begin{tabular}{|r|l|l|r|l|l|l|}
\hline No. & \multicolumn{1}{|c|}{ Test method } & Abbreviation & No. of tests & \multicolumn{1}{|c|}{ Conditions } & \multicolumn{1}{c|}{ Standard } & Reference \\
\hline 1. & Tapping Fork & TF & $5+5$ & dry sample & PN-88/B-04481 & {$[6]$} \\
\hline 2. & Vibrating Table & VT & 10 & dry sample & $\sim$ ASTM D 4253 - 00 & {$[1]$} \\
\hline 3. & Vibrating Hammer & VH & 5 & wet sample & BS 1377-4: 1990 & {$[3]$} \\
\hline 4. & Standard Proctor & SP & 5 & dry sample & PN-88/B-04481 & {$[6]$} \\
\hline 5. & Modified Proctor & MP & 5 & dry sample & PN-88/B-04481 & {$[6]$} \\
\hline 6. & Funnel $d=12.5 \mathrm{~mm}$ & F12 & 9 & dry sample & ASTM D 4254 - 00 & {$[2]$} \\
\hline 7. & Funnel $d=10 \mathrm{~mm}$ & F10 & 7 & dry sample & PN-88/B-04481 & {$[6]$} \\
\hline 8. & Kolbuszewski & K & 10 & dry sample & BS 1377-4: 1990 & {$[3]$} \\
\hline
\end{tabular}

The minimum values of dry density were determined with the use of Funnel method and Inverting Cylinder Kolbuszewski method (the latter one in the version described in British Standard). The volume of the applied cylinder was 1 litre. The Funnel method was applied in two options: ASTM option, where the procedure and the funnel dimensions are described in detail (the funnel is fitted with a $15 \mathrm{~cm}$ long spout, with $12.5 \mathrm{~mm}$ diameter); and the Polish Standard (PN) option - where there is no detailed specification of funnel dimensions, so the applied funnel was $10 \mathrm{~cm}$ long and its diameter was $10 \mathrm{~mm}$. The details relating methods used to determine $\rho_{d \min }$ are presented in Tab.1 (points $6 \div 8$ ). The total number of 26 values of $\rho_{d \min }$ of the tested sand was determined. 


\section{Results and Discussion}

According to the postulate presented in the introductory part, standard methods for the determination of the dry density values of soil should provide considerable efficiency and reproducibility of results. The method efficiency was evaluated against the mean value of the test results. In the case of $\rho_{d \max }$ tests, a method was assumed to be more efficient, when it returned higher values of this parameter, whereas for $\rho_{d \text { mim }}$ test - when it returned lower values of dry density. The method reproducibility was evaluated against the scattering of results, i.e. the difference between the maximum and minimum values of the parameter.

The test results are presented in Fig. 2. Figure 2a refers to the maximum value of dry density. The subsequent bars, put in a growing order, present mean values obtained in the tests. The graph shows that the lowest value of $\rho_{d \max }=1.774$ $\mathrm{g} / \mathrm{cm}^{3}$ was obtained in the Standard Proctor test, whereas the highest value of $\rho_{d \max }=1.899 \mathrm{~g} / \mathrm{cm}^{3}$ - in the Vibrating Hammer test. Value $\rho_{d \max }$ obtained in the VH test exceeds considerably all the remaining results. Evaluating the methods in terms of reproducibility of results, it should be stated that the lowest reproducibility (the highest scattering of results) was obtained for Tapping Fork. As mentioned before, human factor considerably influenced the results, and the test was performed by two laboratory workers. The highest reproducibility was obtained in the Vibrating Hammer test.

a)

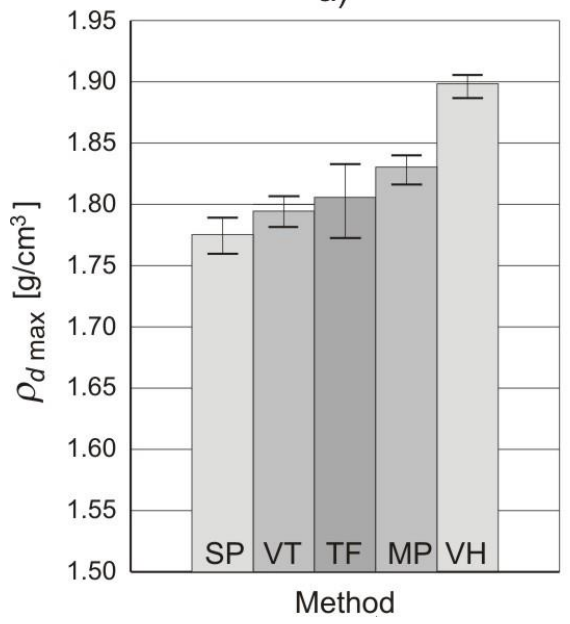

b)

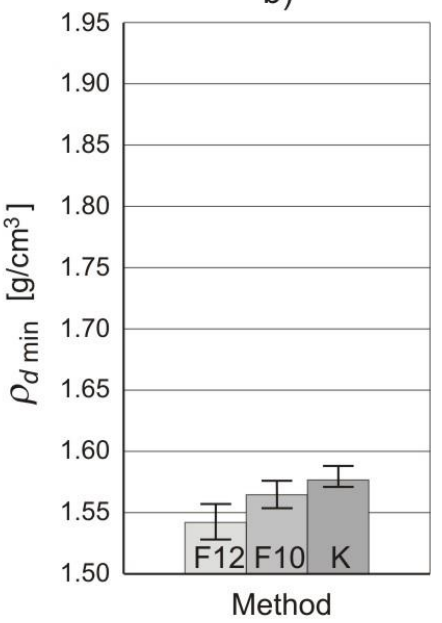

Fig. 2: Mean values and scattering of results for dry density tests: a) $\rho_{d \max }$ b) $\rho_{d \min }$.

Figure $2 b$ presents the results for the minimum values of dry density. In this case, the lowest obtained value equalled $\rho_{d \min }=1.542 \mathrm{~g} / \mathrm{cm}^{3}$ (the highest efficiency of loose arrangement of sand grains) and was obtained with the use of the funnel $\left(12.5 \mathrm{~mm}\right.$ diameter). The highest values of density $\rho_{d \min }=1.577 \mathrm{~g} / \mathrm{cm}^{3}$ (thus, the poorest efficiency) was obtained with the use of Kolbuszewski method. However, it should be stated that F12 method revealed the lowest reproducibility, whereas method $\mathrm{K}$ revealed the highest reproducibility (the scattering of results is the smallest).

Knowing the dry density values for sand, obtained with the particular methods, it is now possible to analyse the index of density. Figure 3a presents the comparison between the values of the index of density according to Polish Standard (PN) and British Standard (BS) in the function of dry density. In order to determine $I_{D(\mathrm{PN})}$, dry density $\rho_{d \min }$ obtained with the F12 method (since standard [6] does not specify the funnel's dimensions), as well as $\rho_{d \max }$ obtained with the TF method were used. In order to determine $I_{D(\mathrm{BS})}$, dry density $\rho_{d \min }$ obtained with the K method, and $\rho_{d \max }$ obtained with the VH method were used. The differences are significantly large. For example, according to Polish Standard (PN), soil with apparent density $\rho_{d}$ $=1.77 \mathrm{~g} / \mathrm{cm}^{3}$ is considered to be very dense $\left(I_{D(\mathrm{PN})}=0.88\right)$, whereas according to British Standard $(\mathrm{BS})-$ its state would be considered as merely medium dense $\left(I_{D(\mathrm{BS})}=0.64\right)$.

Figure $3 \mathrm{~b}$ presents the relative error of the index of density determined with the (PN) method in relation to the (BS)

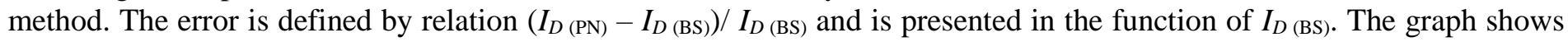
that for soils in the dense state, $I_{D(\mathrm{PN})}$ exceeds $I_{D}$ (BS) by approximately $1 / 3$, on average; within the medium dense state - by more than $40 \%$; within the loose state - by approximately $80 \%$, and finally within the very loose state - even several times. 
a)

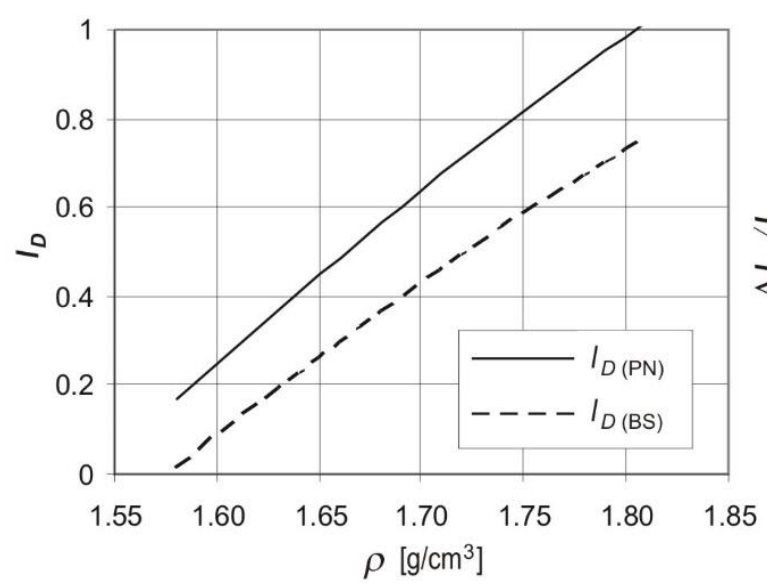

b)

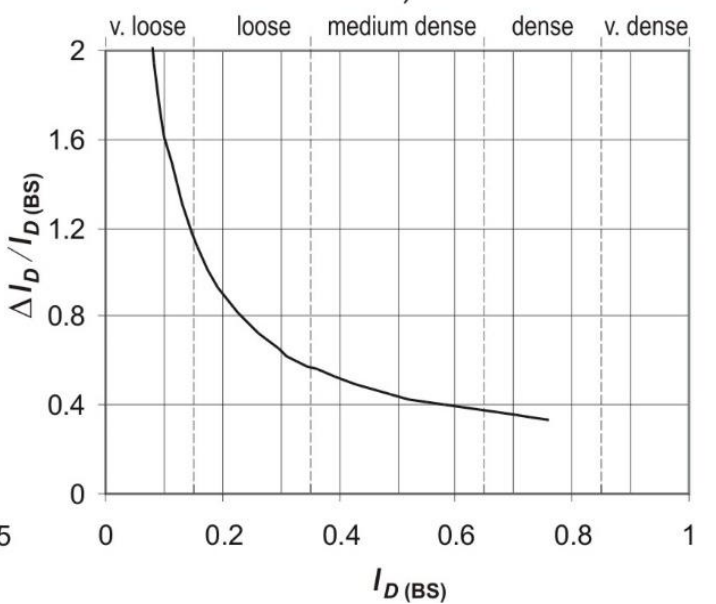

Fig. 3: The index of density for sand according to Polish Standard (PN) and British Standard (BS): a) comparison of values, b) the relative error.

\section{Conclusions}

The results of the performed tests allow drawing the following conclusions referring methods for testing of dry density of sand:

- The Vibrating Hammer method is the most efficient and the obtained results are highly reproducible. However, the procedure requires soil to be vibrated in water, which is cumbersome, and the subsequent desiccation of the samples is time consuming.

- The Modified Proctor method is also an efficient and reproducible method, and could be used for the determination of the index of density of soil. Its additional advantage is the fact that the most geotechnical laboratories all over the world are equipped with the Proctor's apparatus, and the application of this procedure would provide an easy tool for the determination of the relation between relative compaction and index of density (100\% compaction according to Modified Proctor would be equivalent of $I_{D}=1$ ).

- The Tapping Fork method of oven-dry samples should not be used due to its inconsiderable reproducibility.

- Among the methods for testing the minimum dry density, worth mentioning is the Funnel method $(d=12.5 \mathrm{~mm})$ due to its considerable efficiency and the simplicity of the equipment used.

The fundamental conclusions of the performed tests relate the degree of the index of density of soil determined on the basis of standards of individual countries. Significant differences resulting from the comparison of parameter $I_{D}$ calculated according to Polish and British standards suggest the necessity of introduction of additional notation in the symbol which would clearly indicate the methodology used while its determination, e.g. $I_{D}$ (PN) or $I_{D}$ (BS). In the face of globalization, the lack of such additional notations may lead to serious misunderstandings between engineers using various methods.

In noncohesive soils, the index of density is frequently calculated on the basis of in-situ tests, due to the difficulties in obtaining samples of undisturbed structures to determine apparent density $\rho_{d}$. For this purpose, correlations between resistances of the probe insertion (e.g. SPT, DP or CPT) and the index of density are used. The correlation relations elaborated in various countries, based on national procedures for $I_{D}$, determination, may lead to considerably different evaluation of the state of the same soil.

The ultimate solution to the presented problem may be the establishing of international standards for testing the index of density, similarly to the Eurocode 7 standard.

The above conclusions have been drawn on the basis of test of only one type of sand. In order to confirm them it would be necessary to carry out broad studies in various laboratories on different types of soil.

\section{References}

[1] ASTM Standards: D 4253 - 00 Standard test methods for maximum index density and unit weight of soils using a vibratory table. 
[2] ASTM Standards: D 4254 - 00 Standard test methods for minimum index density and unit weight of soils and calculation of relative density.

[3] BS 1377: Part 4: 1990 Soils for civil engineering purposes. Compaction-related tests.

[4] EN 1997-2:2007 Eurocode 7 - Geotechnical design - Part 2: Ground investigation and testing.

[5] U. Smoltczyk, Geotechnical engineering handbook. Vol. 1: Fundamentals. Berlin: Ernst \& Sohn, 2002.

[6] PN-88/B-04481 Engineering soil. Testing of soil samples (in Polish).

[7] T. L. Youd, "Factors controlling maximum and minimum densities of sands," in Evaluation of relative density and its role in geotechnical projects involving cohesionless soils, E. T. Selig and R. S. Ladd, Ed. Baltimore: ASTM STP523, 1973, pp. 98-113.

[8] E. W. Brand, "Some observations on the control of density by vibration," in Evaluation of relative density and its role in geotechnical projects involving cohesionless soils, E. T. Selig and R. S. Ladd, Ed. Baltimore: ASTM STP523, 1973, pp. 121-132.

[9] R. Dobry and R. V. Whitman, "Compaction of sand on a vertically vibrating table," in Evaluation of relative density and its role in geotechnical projects involving cohesionless soils, E. T. Selig and R. S. Ladd, Ed. Baltimore: ASTM STP523, 1973, pp. 156-170.

[10] F. A. Tavenas and P. La Rochelle, "Accuracy of relative density measurements," Géotechnique, vol. 22, no. 4, pp. 549$562,1972$.

[11] A. Prochaska and V. Drnevich, "One-point vibrating hammer compaction test for granular soils," in Proceedings of Geo-Frontiers. Advances in Pavement Engineering, Austin, TX, 2005, pp. 1-15.

[12] Y. Yoshimi and I. Tohno, "Statistical significance of the relative density," in Evaluation of relative density and its role in geotechnical projects involving cohesionless soils, E. T. Selig and R. S. Ladd, Ed. Baltimore: ASTM STP523, 1973, pp. 74-84.

[13] EN-ISO 14688-2:2004 Geotechnical investigation and testing - Identification and classification of soil. Part 2: Principles for a classification.

[14] W. St. Szajna and P. Lechocka, "Evaluation of the two methods of determining dry bulk density of sand: tapping fork test and vibrating table test," Roads and Bridges, Accepted for publication. 